\title{
Last gasps of a black hole
}

\section{Eugenio Bianchi*}

The Pennsylvania State University

E-mail: ebianchi@gravity.psu.edu

After 40 years of active research the question of the fate of information that falls into a black hole is still open [1]. In this talk I will discuss recent results [2] that allow us to compute the entanglement entropy production in black hole evaporation. In particular I present a study of the information release in a model that takes into account the loop quantum gravity resolution of the black hole singularity [3]. The analysis of this phenomenon provides new insights into the entanglement structure of space-time during and after the complete evaporation of the black hole. References

[1] S.W. Hawking, Breakdown of predictability in gravitational collapse, Phys. Rev. D 14 (1976) $10,2460$.

[2] E. Bianchi and M. Smerlak, Entanglement entropy and negative-energy fluxes in twodimensional spacetimes, (2014), arXiv:1404.0602

[3] C. Rovelli and F. Vidotto, Planck stars, (2014), arXiv:1401.6562

Frontiers of Fundamental Physics 14 - FFP14,

15-18 July 2014

Aix Marseille University (AMU) Saint-Charles Campus, Marseille

${ }^{*}$ Speaker. 